\title{
Einfluss der Exsudatsentleerung auf das Eiweiss und den kolloid=osmotischen Druck des Blutes bei Pleuritiden.
}

\author{
(Studien über den kolloid-osmotischen Druck des Bluts \\ im normalen und pathologischen Zustand. XI.) \\ Von \\ Keikichi Nishiyama. \\ (西西 綮吉) \\ (Aus der Medizinisehen Klinit von Prof. Dr. T. Kato, \\ Tohoku Reichsuniversität zu Sendai.)
}

In X. Mitteilung ${ }^{1)}$ vorliegender Studien habe ich darüber berichtet, dass ich bei 16 in aller Hinsicht unter ähnlichen Lebensbedingungen befindlichen Fällen von exsudativer Pleuritis ohne Komplikationen vom Krankheitsbeginn an bis zum völligen Rückgang des Exsudates am ganzen Krankheitsverlauf entlang den Eiweissgehalt und den kolloid-osmotischen Druck sowohl des Blutes wie auch des Exsudates bestimmte, die Schwankungen ihrer Werte beobachtete und auf obige Weise davon überzeugt war, dass zwischen dem spontanen Rückgang. des Exsudates und der Veränderungen des Eiweissgehaltes und des kolloid-osmotischen Drucks eine ungefähr bestimmte Beziehung besteht.

Die Untersuchung in X. Mitteilung. ${ }^{1}$ war nähmlich darauf gerichtet, der Exsudation bei exsudativer. Pleuritis möglichst freien Lauf zu lassen und dabei auftretende Veränderungen des Blutes und des Exsudates durch die wöchentlich einmalige Bestimmung derselben zu beobachten. Im vorliegende Versuch habe ich im stationären Stadium der Krankheit, sofern es nur irgend möglich, das Exsudat auf einmal gänzlich entnommen und im Anschluss daran die Veränderungen des Eiweisses und kolloid-osmotischen Drucks des Blutes wiederholentlich ermittelt, weiterhin habe ich nach Ablauf bestimmter Zeiträume das Eiweiss und den kolloid-osmotischen Druck des von neuem in der Pleurahöhle vorgekommenen Exsudates bestimmt und hierbei erhobene Veränderungen gewürdigt.

1) Nishi y a ma, Tohoku Journ. Exp. Med., 1933, 22, 505. 
Zu Untersuchungen dienten diejenigen Soldaten, die an exsudativer Pleuritis (serofibrinöse Form) erkrankten und welche ähnlich wie in erster Mitteilung hinsichtlich des Lebensjahres, Körperbaues und der Lebensbedingungen miteinander beinahe identisch waren; übrigens war bei diesen Patienten der Krankheitsprozess im Ablauf einiger Wochen nach dem Krankheitsausbruch schon stationär geworden.

Das Exsudat wurde durch Punktion morgens nüchtern entleert, an diesem Morgen liess ich Kranken das Frühstück entbehren, entnahm ihnen in den Zeiträumen vom direkten Anschluss an die erfolgte Punktion bis zum Ablauf von 4 Stunden die Blutprobe zu einigen Malen und zum letzten Mal nach 24 Stunden. Weil das Exsudat aber nicht sofort nach der Entleerung angesammelt ist, entnahm ich die Exsudatprobe im wesentlichen zweimal, und zwar direkt vor therapeutischer Punktion und 24 Stunden nach Beendigung der Punktion und führte die Bestimmung daran aus.

Die Messung der Eiweisses und kolloid-osmotischen Drucks geschah in ganz derselben Weise wie die in früheren Mitteilungen angeführte.

\section{Dienach Exsudatsentleerung aufgetretenen Ver- änderungen des Eiweisses und des kolloid- osmotischen Drucks des Blutes.}

An 9 Fällen von exsudativer Pleuritis habe ich an dem Zeitpunkt, wo der Krankheitsprozess stationär geworden war, also die Exsudatsmengen nicht mehr erhebliche Schwankungen aufwiesen, das Exsudat durch Punktion-nur mit Ausnahme eines Falls-fast vollkommen entleert, und im direkten Anschluss daran habe ich ihnen die Blutprobe einmal, dann weiter dieselbe nach 30 Minuten, einer Stunde, 2, 4 und 24 Stunden, im ganzen also zu 6 Malen entnommen und daran die Eiweissmenge und den kolloid-osmotischen Druck bestimmt und hierbei ermittelte Werte desselben auf den kolloid-osmotischen Druck für 1\% Eiweiss (im folgenden kurz als Druck pro \% bezeichnet) umgerechnet.

Was die Versuchsresultate anbelangt, waren der Eiweissgehalt, der kolloid-osmotische Druck und Druck pro \%, wie aus Tab. 1 zu ersehen ist, im ganzen genommen, durch Punktion vorübergehend herabgesetzt.

Da die Mengen des entleerten Exsudates je nach den Fällen zwischen 280-1530 ccm variierten, habe ich gesamte Falle in 2 Gruppen und zwar in diejenige Gruppe mit weniger als $500 \mathrm{ccm}$ Exsudatsmengen, wie z. B. Nr. 1, 2, 3 und 4, sowie in solche Gruppe mit uiber $600 \mathrm{ccm}$ Exsudatsmengen eingeordnet und die erstere als Gruppe A und die letztere als Gruppe B bezeichnet; die nach Exsudatsentleerung' aufgetretenen Veränderung des Bluteiweisses und kolloid-osmotischen Drucks des Blutes wurden auf prozentuale Werte umgerechnet und diese als dreierlei Kurven von Eiweiss, kolloid-osmotischem Druck und Druck pro \% in Fig. 1 für jede Gruppe gesondert, registriert. 


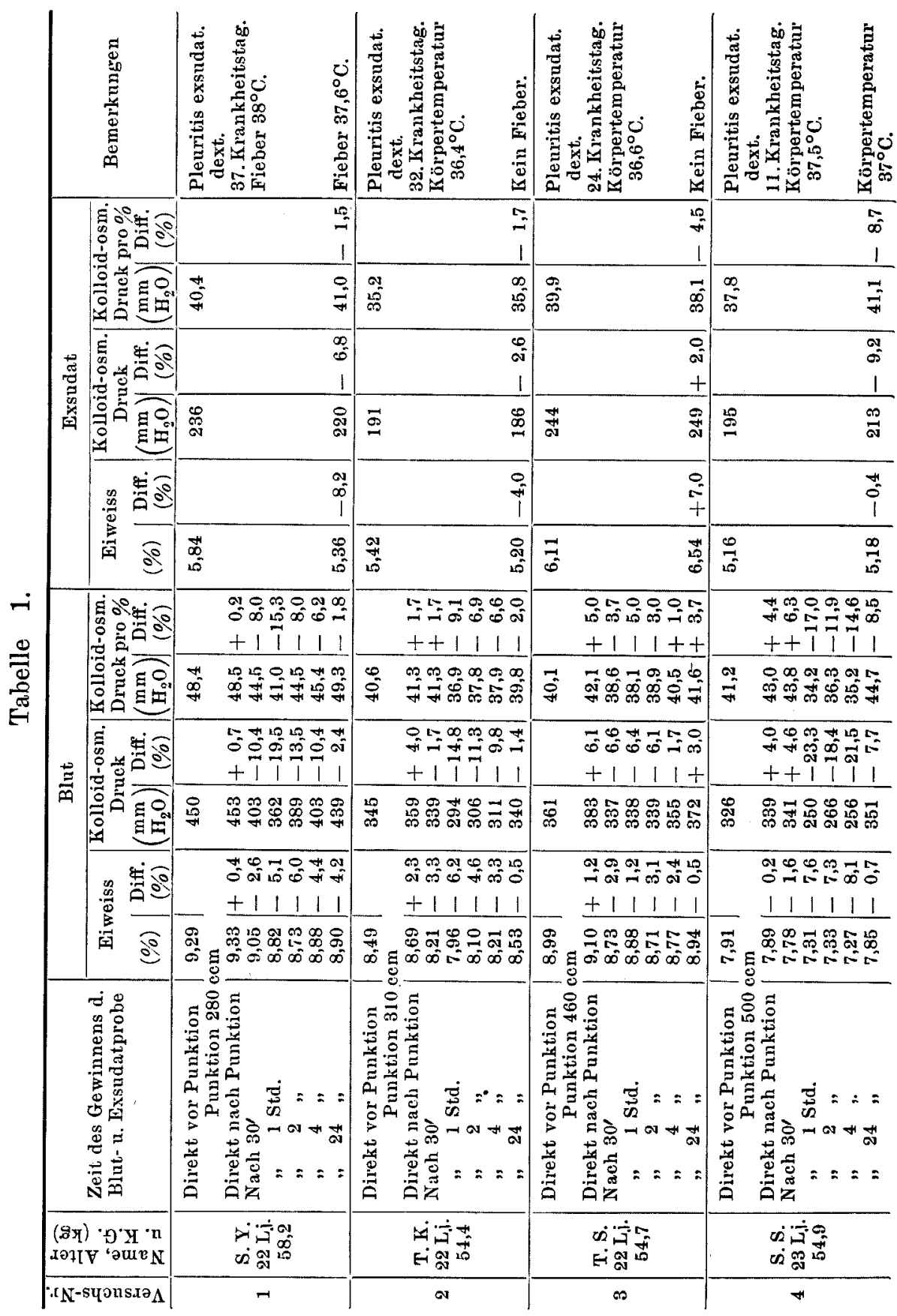




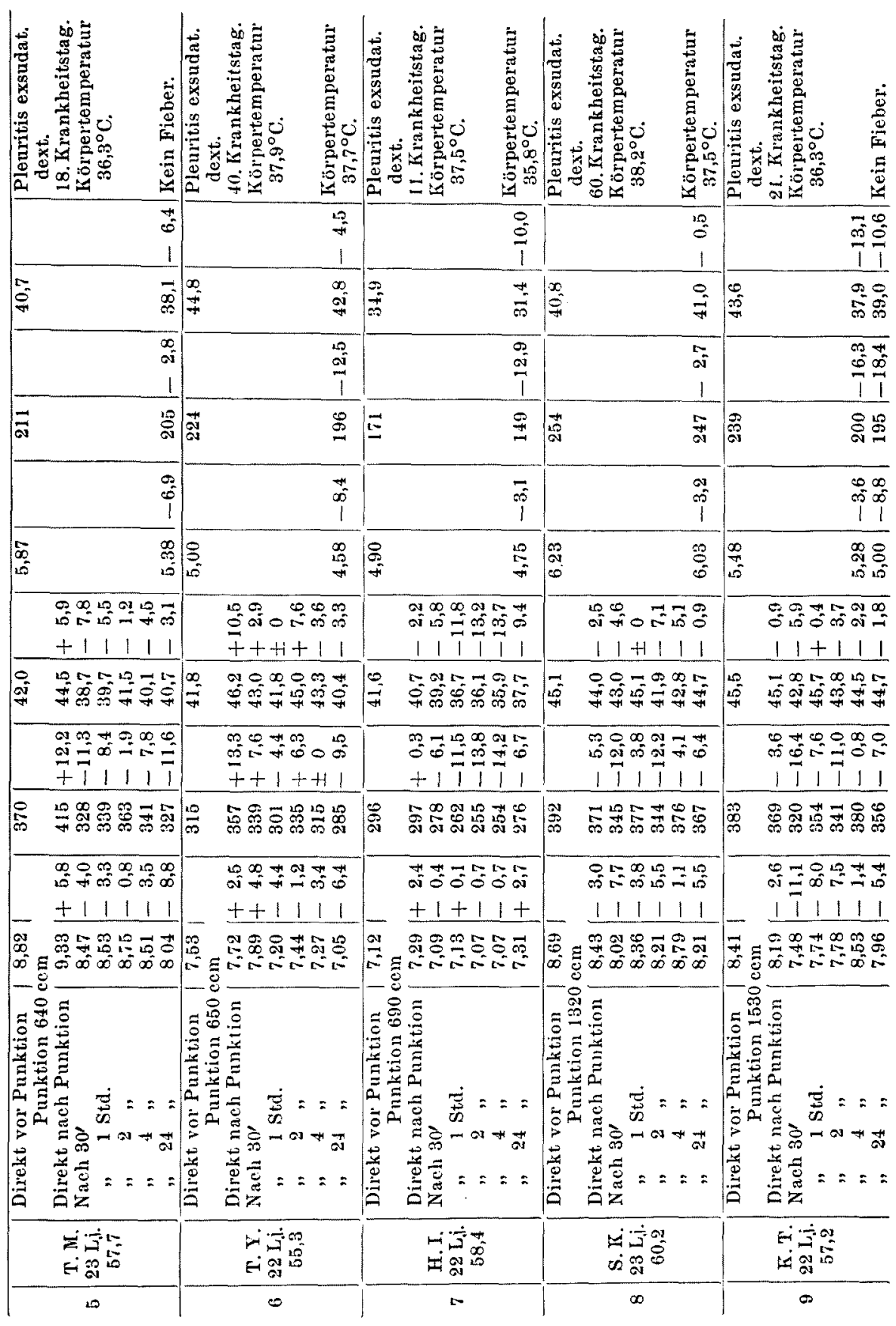



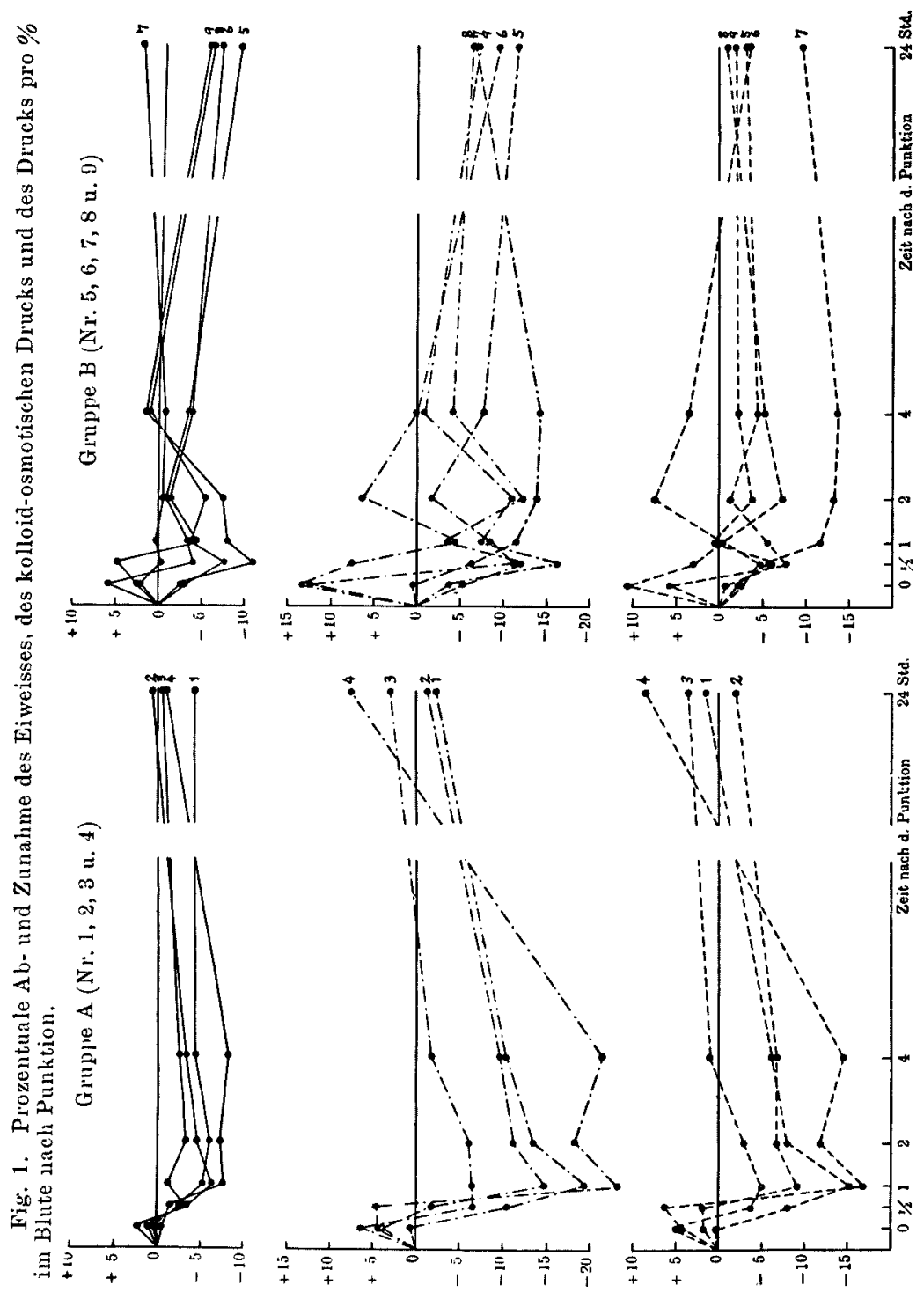

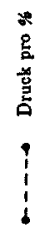
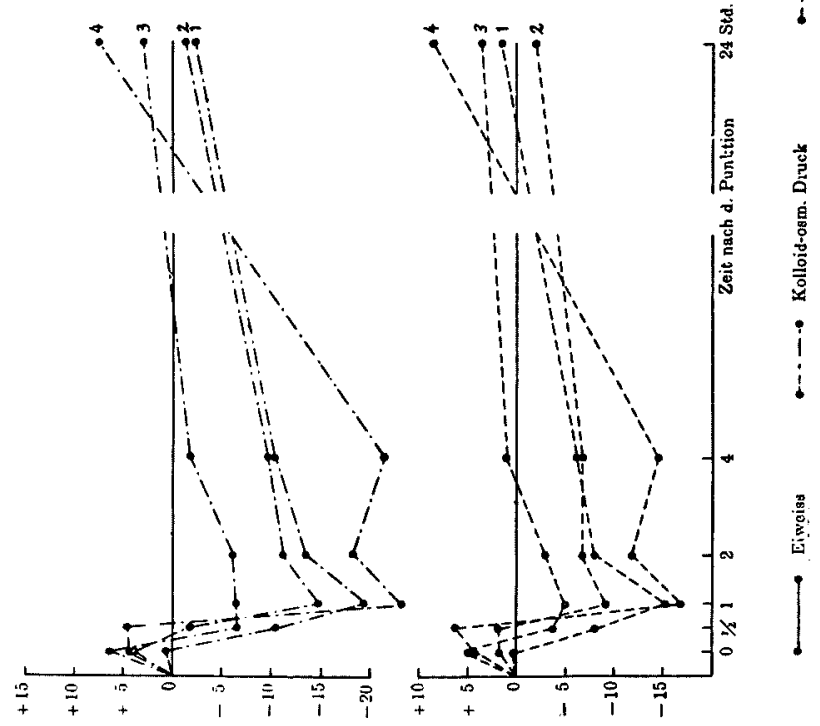

Beim Anblick der Kurve für dic Gruppe A fällt auf, dass der Eiweissgehalt und der kolloid-osmotische Druck kurz nach erfolgter Punktion im allgemeinen mehr oder minder ansteigen, jedoch schon nach 30 Minuten ausschliesslich in erheblichem Masse absinken, in der 1., 2. und 4. Stunde noch mehr deutlich heruntergingen, endlich 
in 24 Stunden beide sich im grossen und ganzen den Anfangswerten nähren. Der Druck pro \% geht auch im wesentlichen den Veränderungen der beiden ersteren parallel, indem er unmittelbar nach Punktion gewissermassen ansteigt, dann bedeutend herabfällt und nach 24 Stunden sich bis auf den annähernden Anfangswert wieder hergestellt.

An der Kurve für die Grpppe B erkennen wir, dass die unmittelbar nach Punktion aufgetretenen Schwankungen des Eiweissgehaltes und des kolloid-osmotischen Drucks in allgemeinen gegenüber der Gruppe A unvergleichbar gross sind, indem sie gleich nach Punktion teils ansteigen, teils herabfallen, bis sie schliesslich aber in der einhalb-, ein-, zwei- oder vierstündigen Blutprobe meistens kontinuierlich, selten aber transitorisch deutlich abfallen. Hier unterscheidet sich die Gruppe B dadurch von der Gruppe A, dass die Kurven in der nach 24 Stunden entnommenen Blutprobe alle (ausgenommen einen Fall, Nr. 7, bezüglich des Eiweisswertes) auf herabgesetzten Werten beharren und nie auf Anfangswerte zurückkehren wollen.

Also bestanden bei der Gruppe B die Veränderungen zwar im Absinken des Eiweissgehaltes und des kolloid-osmotischen Drucks, die beiden aber fielen darauf allmählich herab, indem sie starke Schwankungen aufwiesen, und konnten sich nicht mehr selbst nach 24 stündigem Ablauf zu Anfangswerten wiederherstellen. Beim Druck pro $\%$ liess sich auch beinahe dasselbe Verhältnis erkennen.

Kurzum, wir können dieses Phänomen dahin deuten, dass bei relativ geringerer Exsudatsentleerung (man könne auch dafür den Ausdruck ,,bei geringerer Exsudatsansammlung" gebrauchen, weil man das betreffende Exsudat völlig entleerte), der Eiweissgehalt und der kolloid-osmotische Druck unmittelbar nach Punktion nach initialer unerheblicher Steigerung mit gleichem Schritt_auch der Druck pro $\%$ ihnen auf Tritt und Schritt folgend-beträchtlich herabgesetzt und nach 24 Stunden im grossen und ganzen bis nahe an frühere Werte wiederhergestellt waren, während bei relatjv grösserer Exsudatsentleerung bzw. Exsudatsansammlung die Kurven mehrere Stunden lang nach Punktion unter ziemlich starken Schwankungen allmählich abfielen und so auch selbst in der 24 stündigen Blutprobe nicht mehr bis auf frühere Werte wiclerhergestellt werden konnten.

In X. Mitteilung habe ich die Veränderungen des Bluteiweisses und des blutkolloid-osmotischen Drucks in den Zeiträumen vom Krankheitsbeginn bis zum spontanen, dem Verlauf gemäss erfolgten Rückgang des Exsudates verfolgt. In vorliegender Mitteilung wurde die Untersuchung in den Zeiträumen vom Zeitpunkt an, wo das betreffende Exsudat künstlich vollkommen entleert worden war, bis zu wieder auftretender Exsudatsansammlung durchgeführt. Von höchstem In- 
teresse wäre es, so gewonnene Resultate mit den Resultaten in $\mathbf{X}$. Mitteilung vergleichend zu würdigen.

Wenn man bei exsudativer Pleuritis zu einer Zeit, wo der Krankheitsprozess sich unter der Exsudatsansammlung im stationären $\mathrm{Zu}$ stand befindet, das Exsudat auf einmal entleert hat, so stellt sich, solange die entzündliche Veränderung der serösen Membran in der Pleurahöhle noch nicht vollkommen geheilt ist, wieder sofort die Exsudatbildung ein, worauf schon $\mathrm{S} \mathrm{ch}$ a de und seine Mitarbeiter ${ }^{2)}$ hingewiesen haben. Bei 9 Fällen von mir untersuchten Pleuritiden habe ich mich auch davon überzeugt, dass bei ihnen Exsudate schon mehrere Stunden nach Punktion tatsächlich wieder auftraten. Die nach erfolgter Punktion auftretenden Veränderungen des Bluteiweisses und des blutkolloid-osmotischen Drucks können also dahin erklärt werden, dass zu einer Zeit, wo der Krankheitsprozess sich im stationären Zustand befindet, der kolloidale Zustand zwischen dem Blut und Exsudat aufrecht erhalten wird, dieser Gleichgewichtszustand durch die Beseitigung des Exsudates gestört wordon ist und die Eiweissteilchen des Blutes durch die entzündlich veränderte, mehr permeabel gewordene Pleura hindurch rasch in die Pleurahöhle ubergehen. Die unmittelbar nach Punktion vorübergehend auftretende Zunahme dürfte wohl auf psychischer Beeinflussung des betreffenden Individuums durch den Eingriff beruhen. In Anbetracht, dass bei derartig aufs neue eintretender Exsudatbildung das Bluteiweiss und der blutkolloid-osmotische Druck deutlich absinken, lässt sich die Tatsache leicht verstehen, dass im Stadium der Exsudatsbildung bei exsudativer Pleuritis das Bluteiweiss und der blutkolloid-osmotische Druck, wie ich es in erster Arbeit gesehen habe, im allgemeinen niedrigere Werte darbieten und mit dem herannahenden Aufhören der Exsudation wieder ansteigen.

Wenn die Exsudatsansammlung geringfügig und dementsprechend die entleerte Exsudatsmenge spärlich ist, wie es bei der Gruppe A der Fall war, dann ist mehrere Stunden nach erfolgter Punktion das Gleichgewicht $\mathrm{zwischen}$ dem Blut und dem aufs neue vorkommenden Exsudat erhalten, wodurch beide Komponenten schon nach 24 Stunden bis zu ihren früheren Werten wiederhergestellt werden können. Und andererseits, wenn die Exsudatsansammlung intensiv stattfindet und demgemäss auch entlecrte Exsudatsmenge sehr gross sind, wie es bei Gruppe B der Fall war, dann können das Bluteiweiss und der blutkolloid-osmotische Druck, indem das von neuem vorkommende Exsudat kaum stationär wird und dadurch das Gleichgewicht zwischen dem Blut und Exsudat noch nicht vollkommen erhalten werden kann,

2) Schade, Claussen, Häblè, Hoff, Mochizuki u. Birner, Ztschr: f.d. ges. exp. Med., 1926, 49, 334 . 
selbst nach 24stündigem Ablauf nicht auf ihre frühere Werte zurückkommen.

Ich habe Pleuritikern das Exsudat, welches nach Punktion in 24stündigem Ablauf von neuem angesammelt war, durch Punktion entnommen und daran den Eiweissgehalt und den kolloid-osmotischen Druck bestimmt. Diese Bestimmung ergab folgendes: In 7 von 9 Fällen zeigten der Eiweissgehalt und der kolloid-osmotische Druck im Vergleich mit den unmittelbar vor Punktion ermittelten Werten niedrigere Werte, nur in 2 Fällen wiesen sie etwas höhere Werte auf. Es muss also angenommen werden, dass im Exsudat, welches nach erstmaliger Punktion von neuem eintritt, der Eiweissgehalt und der kolloid-osmotische Druck im allgemeinen gegenüber den Werten vor Punktion niedrigere Werte zeigen.

II. Veränderungen des Eiweisses und kolloidosmotischen Drucks des Blutes bei der Infusion der physiologischen Kochsalzlösung in die Pleurahöhle unmittelbar nach Entleerung des Exsudates in soviel Mengen, wie das entnommene Exsudat, sowie Veränderungen der Konzentration und des kolloidosmotischen Drucks der Eiweisskörper, welche in der eingeführten Kochsalzlösung von neuem erscheinen.

Im vorigen Kapitel sind angeführ't worden die Beobachtungen über die Veränderungen des Eiweisses und des Kolloid-osmotischen Drucks des Blutes bei abermals beginnender Exsudation, die sich in der vorher durch Punktion fast völlig leer gewordenen Pleurahöhle wieder zum Vorschein kommt sowie über die Veränderungen derselben des von neuem gebildeten Exsudates.

In vorliegender Untersuchung kam es darauf an, nachdem ich in die zuvor durch Punktion entleerte Pleura, anstatt diese im leeren Zustand zu belassen, die physiologische Kochsalzlösung in denjenigen Mengen, die den Mengen des vorher entleerten Exsudates gleichkamen, eingeführt hatte, folgende Verhältnisse zu analysieren: In welcher Weise das Bluteiweiss und der blutkolloid-osmotische Druck durch diesen Eingriff beeinflusst werden könnten, inwieweit das Eiweiss dann in der Kochsalzlösung zunehmen würde und welches Verhalten der kolloid-osmotische Druck desselben zeigen würde, wenn voraussichtlich die eingeführte Kochsalzlösung allmällich resorbiert wird, und wenn gleichzeitig mit dieser Resorption die Exsudation sich erneut einstellt.

Es wurde die Untersuchung an 4 fieberfreien im relativ stationären Krankheitszustand befindlichen und doch mit reichlicher Exsudatsansammlung be- 
hafteten Pleuritikern durchgeführt. Der Versuch wurde stets frühmorgens nüchtern vorgenommen, am Morgen des Versuchs wurde kein Frühstück dargereicht. Die Versuchperson lag auf dem Bett auf erkrankter Seite; nachdem man die in dieser Stellung für ca. eine Stunde die Ruhe befolgen liess, nahm man mittels eines $Y$ - förmigen Troikarts sehr langsam die Entleerung des Exsudats vor und infundierte dann, wenn die entleerte Exsudatsmenge $1500 \mathrm{ccm}$ erreicht hat, die zuvor steril bereitgestellte, auf $38^{\circ} \mathrm{C}$ erwärmte physiologische Kochsalzlösung um eben soviel Menge, als das Exsudat entleert worden war, in gleichseitige Pleurahöhle, damit wurde die Operation beendet. Hierbei empfing der Patient subjektiv keine Beschwerden.

Da die zuvor vorhanden gewesenen Exsudatsmengen in jedem von 4 Fällen jeweils über $1500 \mathrm{ccm}$ waren, stellte die tatsïchlich in der Pleurahöhle zurückgebliebene Flüssigkeit eine Mischung von der physiologischen Kochsalzlösung und dem restierten Exsudat dar; kurz im Anschluss an die Operation befand sich das Exsudat, wie aus Tab. 2, auf der die Untersuchungsresultate niedergelegt sind, ersichtlich ist, in sehr stark verdünnter Konzentration, die Pleuraflüssigkejt hierbei war deshalb in Wirklichkeit zum grossen Teil mit physiologischer Kochsalzlösung besetzt. In der Pleurahöhle befindlichen Flüssigkeit nahm jedoch das Eiweiss mit dem Zeitablauf allmählich immer mehr zu, so dass in 5-7 Tagen der Eiweisswert in der Pleuraflüssigkeit ungefähr bis auf den Eiweisswert im Exsudate vor der Einführung der Kochsalzlösung wiederhergestellt wurde.

In Rücksicht darauf wurden hier die Blut- und Exsudatsentnahme in zeitlich anderer Weise als im vorigen Kapitel ausgeführt, indem die Blut- und Exsudatsentuahme einmal unmittelbar vor Operation, einmal im unmittelbaren

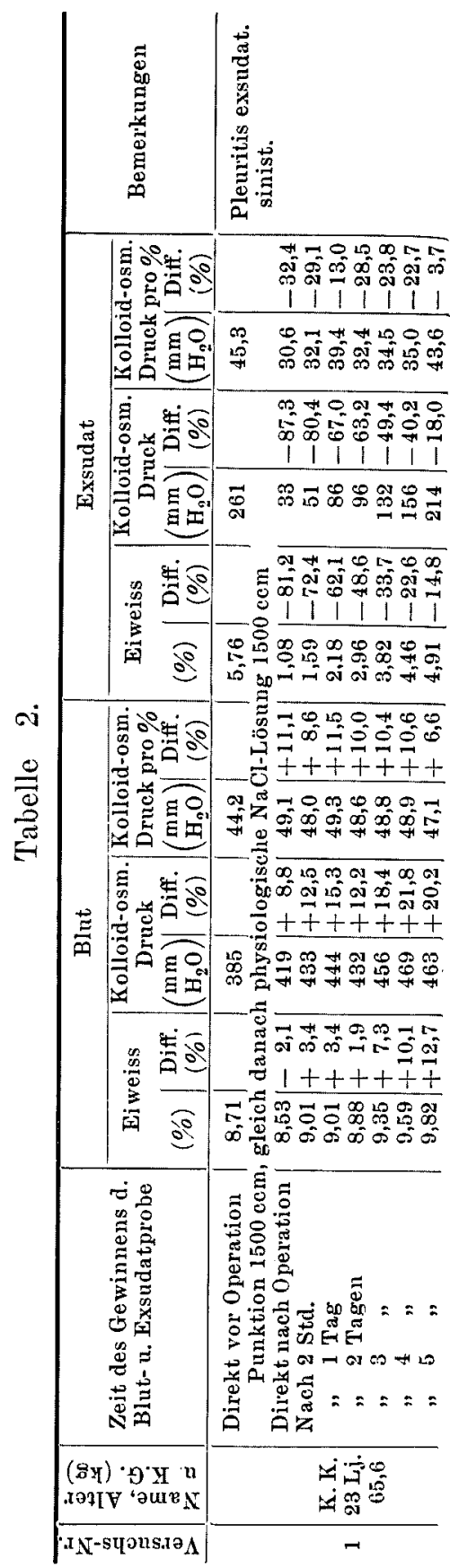




\begin{tabular}{|c|c|c|c|}
\hline & 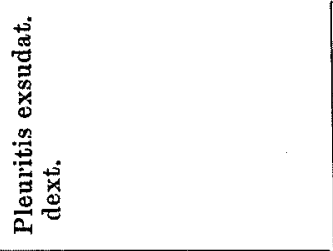 & 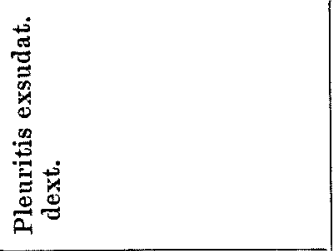 & 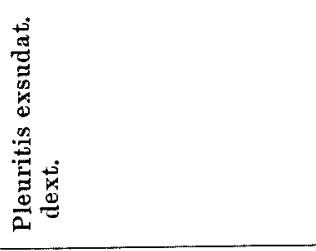 \\
\hline $\begin{array}{l}\overline{00} \\
00 \\
11 \\
11\end{array}$ & 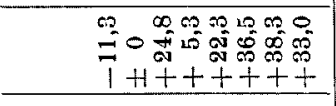 & 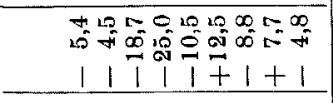 & 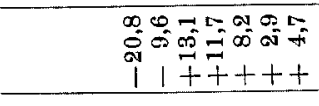 \\
\hline फ़्্ & 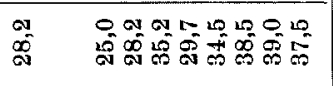 & 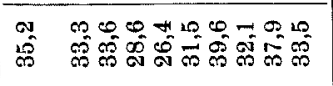 & 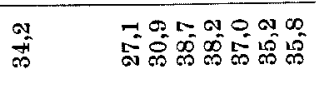 \\
\hline 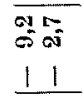 & 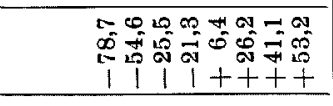 & 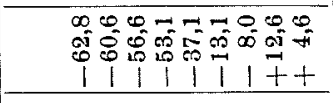 & 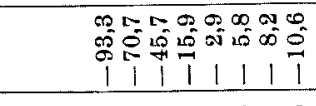 \\
\hline 商落 & 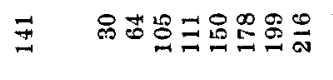 & 농 & 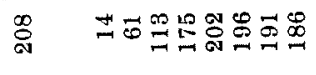 \\
\hline $\begin{array}{l}\infty= \\
\infty= \\
++\end{array}$ & 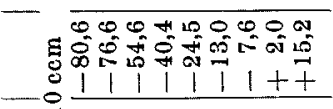 & 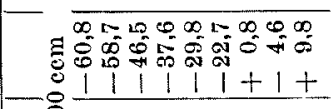 & 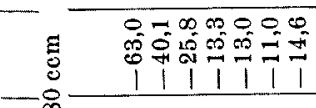 \\
\hline$\overline{0}$ & 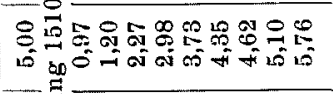 & 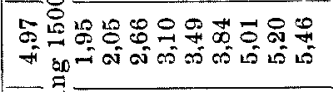 & 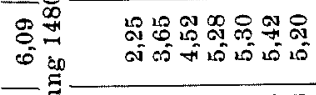 \\
\hline $\begin{array}{l}0= \\
0=0 \\
++ \\
\end{array}$ & 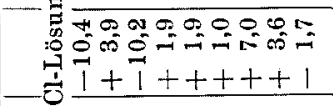 & 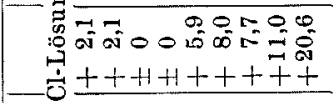 & 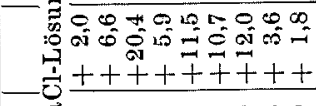 \\
\hline 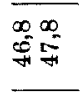 & 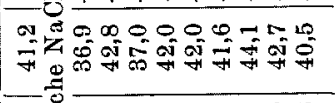 & 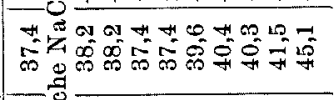 & 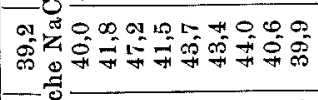 \\
\hline 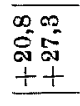 & 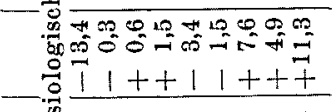 & 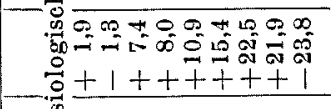 & 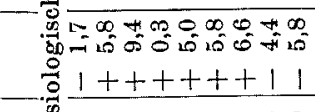 \\
\hline 㝵吕 & 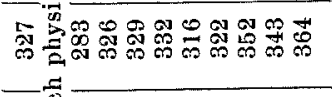 & 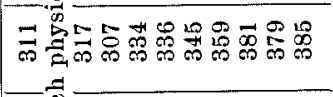 & 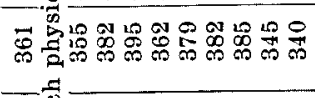 \\
\hline $\begin{array}{l}\overline{0} \\
+5 \\
+1 \\
+1\end{array}$ & 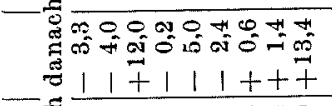 & 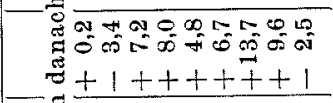 & 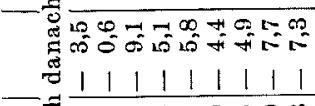 \\
\hline 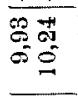 & 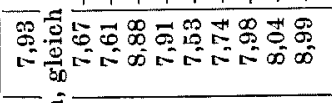 & 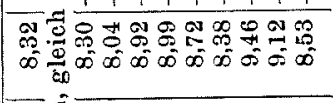 & 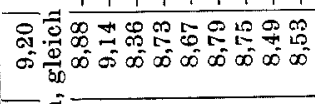 \\
\hline \multirow[t]{3}{*}{$\begin{array}{l}== \\
\text { Or } \\
==\end{array}$} & 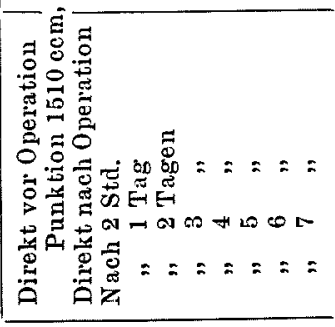 & 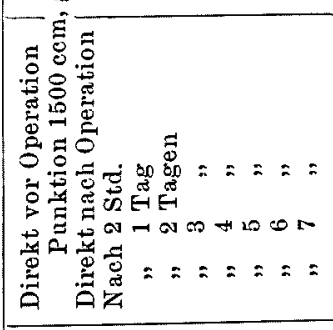 & 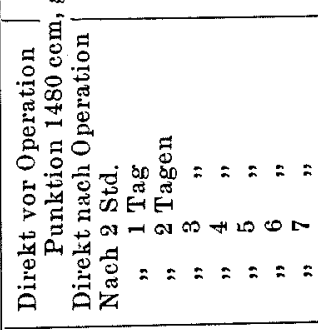 \\
\hline & 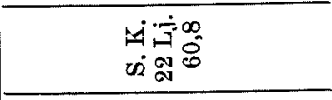 & 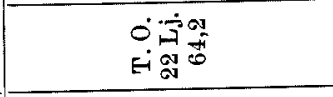 & 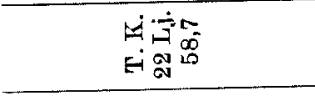 \\
\hline & a & $m$ & $H$ \\
\hline
\end{tabular}



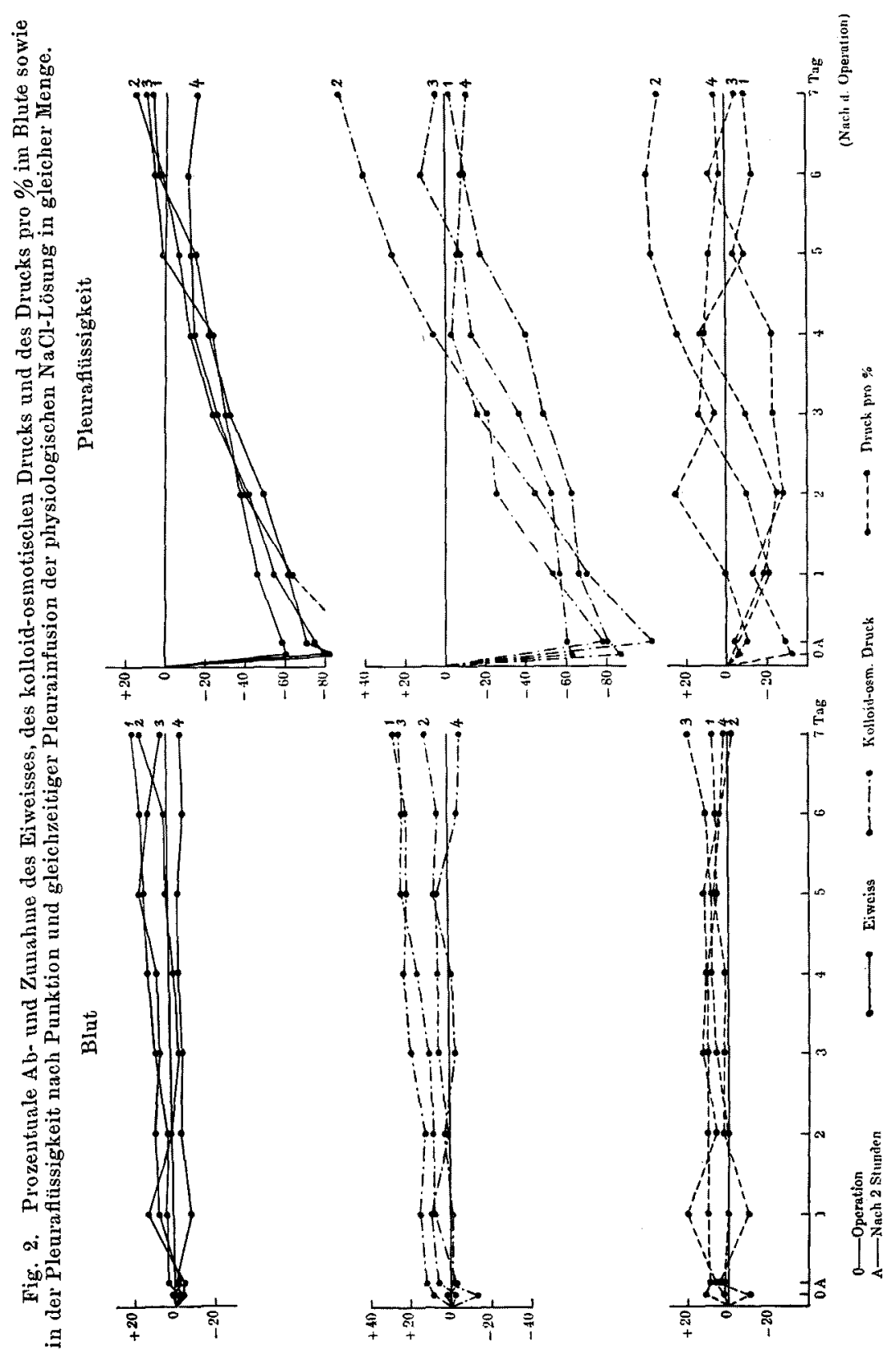
Anschluss an die beendete Infusion physiologischer Kochsalzlösung und einmal in 2 Stunden darauf, also dreizeitig vorgenommen wurde, ohne dass die weitere Entnahme an demselben Tage stattfand. Weiter wurden die Blut- und Exsudatprobe einmal täglich, morgens nüchtern, vom darauf folgenden Tag an bis zum siebten Tage fortgesetzt entnommen.

Die Versuchsresultate sind ausschliesslich auf die Tab. 2 aufgetragen, der Eiweissgehalt, der kolloid-osmotische Druck und Druck pro \% sowohl des Blutes als auch des Exsudates in dreierlei Kurvenformen in Fig. 2 angegeben.

Zunächst wollen wir die Veränderungen im Blut streifen. Wie aus der Tabelle, besonders aber aus Kurven ersichtlich ist, waren das Eiweiss, der kolloid-osmotische Druck und der Druck pro \% während des 24stündigen Verlaufes nach Punktion und Kochsalzinfusion nicht übereinstimmend herabgesetzt, wie bei einfacher Punktion im vorigen Kapitel, im Gegensatz hierzu waren in 3 von 4 Fällen sowohl das Eiweiss wie auch der kolloid-osmotische Druck eher erhöht und beharrten im grossen und ganzen im Verlauf von 7 Tagen auf ihrem erhöhten Zustand, ohne inzwischen erhebliche Schwankungen aufgewiesen zu haben. Der Druck pro \% schwankte nach oben und unten bis zum Ablauf von 24 Stunden nach Kochsalzeinführung, vom zweiten Tage an verblieb er aber im allgemeinen an etwas erhöhtem Zustand und verlief weiter ohne erhebliche Schwankung.

Was die Pleuraflüssigkeit anbelangt, war sie, obwohl der darin enthaltene Eiweissgehalt sich besonders im unmittelbaren Anschluss an die Infusion physiologischer Kochsalzlösung je nach den noch in der Pleurahöhle zurückgebliebenen Exsudatsmengen verschieden verhielt, im grossen und ganzen in 5- bzw. 3 fach verdünter Konzentration, wobei auch der kolloid-osmotische Druck einen sehr niedrigen Wert anzeigte. Vor allem aber bei Nr. 4 ist das Eiweiss der Pleuraflüssigkeit im unmittelbaren Anschluss an die Infusion der Kochsalzlösung und nach 2 Stunden darauf fast unmessbar geworden.

In eingeführter Kochsalzlösung aber war die Eiweisskonzentration inzwischen gesteigert, so dass diese am 5 . bzw. 7. Tage nach Kochsalzeinführung bis nahe an den Eiweisswert im vor Punktion vorhanden gewesenen Exsudat fast in gerader Linie zunahm. Und der kolloid-osmotische Druck nahm im wesentlichen parallel mit dem Eiweiss zu, und zwar bei Nr. 2 ging die Vermehrung des kolloid-osmotischen Drucks mit schnellem Schritt vonstatten und nach 4. Tage in noch erheblicherem Masse. Bei Nr.1 erfolgte die Vermehrung desselben relativ langsam.

Auf den Druck pro \% bezogen, zeigte dieser deshalb bei Nr. 2 im allgemeinen einen erheblich höheren Wert, während er bei $\mathrm{Nr}$. 1 hingegen einen niedrigeren Wert aufwies, und bei Nr. 3 und 4 hat er bis 
zum 3. Tage beim niedrigen Wert verweilt, ist danach beinahe bis auf denjenigen Wert, der dem kolloid-osmotischen Druck des vor Punktion vorhanden gewesenen Exsudates entspricht, aufgeschwungen.

Wirft man nun einen Rückblick auf das Elscheinen der Eiweissstoffe in eingeführter Kochsalzlösung, so stellt sich heraus, dass der Eiweisswert mit der Zeit beinahe linienförmig zunimmt und am 5 . bzw. 6. Tage die Eiweisskonzentration des vor Punktion vorhanden gewesenen Exsudates erreicht. Der kolloid-osmotische Druck des in eingeführter Kochsalzlösung enthaltenen Eiweisses steigt im grossen und ganzen parallel mit vermehrter Konzentration des betreffenden Eiweisses auf, in den einzelnen Fällen nimmt er aber bald schneller, bald langsamer gegenüber der Eiweisszunahme zu, wodurch der Druck pro \% nicht immer eine bestimmte Richtung darbietet.

Dies hat wohl dem Umstand zu verdanken, dass je nach Intensität und Extensität der Erkrankung der serösen Membran in der Pleurahöhle diese seröse Haut sich für das Passieren der Eiweissteilchen in verschiedener Weise verhält.

Was die innerhalb der ersten 24 Stunden aufgetretenen Veränderungen des Eiweisses und des kolloid-osmotischen Drucks im Blut anbelangt, sind die beiden im Gegensatz zum Fall, wo das Exsudat lediglich entleert wurde, trotzdem grosse Mengen Exsudat von $1500 \mathrm{ccm}$ entnommen worden waren, dank der Einführung gleicher Mengen physiologischer Kochsalzlösung nicht erniedrigt, sondern vielmehr erhöht und beharren weiter im darauffolgenden Verlauf im allgemeinen auf relativ höheren Werten. Ein derartiges Phänomen dürfte wahrscheinlich wie folgt erklärt werden: Dort, wo man grosse Mengen Exsudats aus der Pleurahöhle entnommen und diese im leeren Zustand belassen hat, wie es in vorigen Kapitel der Fall war, kommt es in der leer gewordenen Pleurahöhle zu rascher Exsudatsbildung, hierbei mag das Blut sein Eiweiss sofort an das Exsudat abgeben und doch dabei kann der Ersatz dafür von seiten des Eiweissdepots im Organismus dem Blut nicht hinreichend angeboten werden, es tritt dann möglicherweise das Absinken des Eiweissspiegels und des kolloidosmotischen Drucks ein; solange aber der Krankheitsprozess sich im mehr stationären Zustand befindet und ein bestimmtes Gleichgewicht zwischen dem Blut und Exsudat erhalten ist, ist etwaige weitere schnelle Exsudatsbildung, selbst wenn grössere Mengen Exsudats entleert worden waren, von vornherein ausgeschlossen, falls die isotonische $\mathrm{NaCl}$-Lösung in gleichen Mengen als wie die vorher entleerte Exsudatsmengen sogleich infundiert worden ist. Weil aber in unmittelbarem Zeitpunkt vor Punktion und Kochsalzeinführung ein Gleichgewicht zwischen Blut und Exsudat bestanden hat, dürfte das Er- 
scheinen der Eiweisskörper in eingeführter NaCl-Lösung folgendermassen erklärt werden.

Es ist zunächst daran denkbar, dass in eben demselben Masse, als die eingeführte Kochsalzlösung allmählich durch die seröse Membran hindurch in das Blut resorbiert worden ist, die Exsudation in die Pleurahöhle stattfinden kann. Es vollziehen sich also hierbei zwischen dem Blute und der in der Pleurahöhle befindlichen NaCl-Lösung zweierlei Austauschvorgänge: Bei dem einen wird die eingeführte Salzlösung vermöge des dem Blut zukommenden kolloid-osmotischen Drucks ins Blut aufgefangen. Bei dem anderen handelt es sich um die vermöge desFiltrationsdrucks durch die erkrankte, mehr permeabel gewordene seröse Haut hindurchgehende eiweissreiche Exsudation in die Kochsalzlösung in der Pleurahöhle.

Dass das Exsudat hierbei im Gegensatz zu demselben, das im vorigen Kapitel erwähnt worden ist, überaus langsam in die Pleuraflüssigkeit übertritt, geht aus dem Umstand offenbar hervor, dass das in der Pleuraflüssigkeit enthaltene Eiweiss selbst im Ablauf der ziemlich längeren Zeiträume (5-7 Tage) linienförmig und allmählich ansteigt.

Beim derartig langsam stattfindenden Übergang des Eiweisses in die Pleurahöhle wäre allerdings zu erwarten, dass die bluteiweissbildende oder-regulierende Funktion des Organismus sich höchstwahrscheinlich in vollkommener Weise vollziehen kann und somit auch vom Eiweissdepot osmoaktive Eiweisskörper reichlich ersetzt werden können, demzufolge das Bluteiweiss nicht abzunehmen braucht und dessen osmotischer Druck eher erhölnt ist.

\section{Zusammenfassung.}

An Kranken mit exsudativer Pleuritis, und zwar bei einem stationären Zustand des Krankheitsprozesses

1. Wenn das Exsudat in grossen Mengen (Mengen, die soweit es mir nur erlaubt war, entleert werden konnten) durch Punktion entnommen worden ist, waren im Blute Eiweissgehalt, kolloid-osmotischer Druck sowie Druck pro \% im allgemeinen transitorisch erniedrigt.

Bei kleineren Mengen des beseitigten Exsudates (also auch bei geringerer Ansammlung des Exsudates) war dieses Absinken in 24stündigem Ablauf wieder hergestellt. Bei grösseren Mengen entnommenen Exsudates (also auch bei reichlich angesammeltem Exsudate) pflegten Eiweiss und kolloid-osmotischer Druck noch in 24 Stunden auf erniedrigtem Zustand zu beharren.

2. Eiweiss und kolloid-osmotischer Druck desjenigen Exsudates, welches nach Ausführung der Punktion von neuem angesammelt war, 
wiesen im allgemeinen niedrigere Werte als dieselben des zuvor vorhanden gewesenen Exsudates auf.

3. Bei dem Verfahren, wo man bei intensiver Exsudatsansammlung grosse Mengen Exsudat (ca. $1500 \mathrm{ccm}$ ) entnommen hatte und in unmittelbarem Anschluss daran die physiologische NaCl-Lösung gleicher Menge in die Pleurahöhle infundiert hat, waren Bluteiweiss und dessen kolloid-osmotischer Druck im allgemeinen nicht erniedrigt, sondern eher erhöht.

4. In der NaCl-Lösung, welche in die durch Punktion entleerte Pleuraböhle eingeführt worden ist, nahm Eiweiss allmählich schubweise zu, bis dasselbe am 5. bzw. 7. Tage nach Kochsalzinfusion annähernd dieselbe Konzentration als wie die Konzentration des Eiweisses des zuvor vorhanden gewesenen Exsudates erreichte. Die Zunahme des kolloid-osmotischen Druckes ging im wesentlichen der des Eiweissgehaltes parallel, in jedem einzelnen Fall war dies jedoch nicht immer der Fall. Mithin war der Druck pro \% ziemlich stärkeren Schwankungen unterworfen.

Zum Schluss ist es meine angenehme Pflicht, Herm Dr. F. Nakaza wa, an hiesiger Klinik, welcher mich bei dieser Arbeit mit Tat und Rat unterstutzte, meinen herzlichen Dank auszusprechen. 